www.jmscr.igmpublication.org

Index Copernicus Value: 79.54

ISSN (e)-2347-176x ISSN (p) 2455-0450

crossref DOI: https://dx.doi.org/10.18535/jmscr/v7i4.190

Journal Of Medical Science And Clinical Research

\title{
Comparison of the Intraperitoneal Instillation of Bupivacaine (0.25\%) and Ropivacaine $(0.25 \%)$ for Postoperative Analgesia in Elective Caesarean Section under Spinal Anaesthesia: A Prospective Randomised Controlled Study
}

\author{
Authors \\ Dr Anita Pareek ${ }^{1}$, Dr Richa Soni ${ }^{2 *}$, Dr Aditya ${ }^{3}$, Dr Pramila Soni ${ }^{4}$
}

Department of Anaesthesia, Sardar Patel Medical College \& AGH, Bikaner, Rajasthan

*Correspondence Author

Dr Richa Soni

Resident, Department of Anaesthesia, Sardar Patel Medical College \& AGH, Bikaner, Rajasthan

Email: richasoni196@yahoo.com

\begin{abstract}
Objective and Aim: Post-caesarean section pain is associated with significant morbidity. Intraperitoneal instillation of local anaesthetics has been a promising technique to minimise the postoperative pain. The aim of our study was to evaluate the relative efficacy of Bupivacaine and Ropivacaine for postoperative analgesia in elective caesarean section in relation to duration of postoperative analgesia and complications. Materials \& Methods: In this study, 90 patients of ASA Grade I and II undergoing elective caesarean section were divided into 3 groups after randomization to receive intraperitoneal instillation of $30 \mathrm{ml}$ Normal saline $0.9 \%$ in group C, $30 \mathrm{ml}$ Bupivacaine $0.25 \%$ in group B and $30 \mathrm{ml}$ Ropivacaine $0.25 \%$ in group $R$ at the end of uterine closure. All patients were assessed for postoperative pain using VAS score at 0, 2,4,6,12 and $24 \mathrm{hrs}$. Rescue analgesic was given when VAS score was $\geq 6$. The timing of the first dose of rescue analgesic and the total requirement of rescue analgesia in the first $24 \mathrm{hrs}$ was noted.

Results: The time for the first rescue analgesic requirement was significantly longer in group $R(7.28 \mathrm{hrs})$ compared to group B(4 hrs) and group C $(2.5 \mathrm{hrs})$ with $p$ value of 0.001 . The total consumption of rescue analgesic drug was more in group $C(275 \mathrm{mg})$ and group B(172mg) as compared to group $R(92 \mathrm{mg})$ with $p$ value of 0.0001 .

Conclusion: We conclude that when compared to bupivacaine, intraperitoneal instillation of Ropivacaine not only provides superior analgesia but also reduces the total rescue analgesic dose consumption without any significant adverse effects in an elective caesarean section.

Keywords: Intraperitoneal instillation, Bupivacaine, Ropivacaine, postoperative analgesia.
\end{abstract}

\section{Introduction}

Child birth is an emotional experience for woman and her family too. Caesarean section is the most common surgery which is performed worldwide and delivery by caesarean section is becoming more frequent. It is estimated that $33 \%$ prevalence is there of caesarean section in India. Pain is ranked highest among undesirable outcomes associated with caesarean section ${ }^{1}$. Acute pain following delivery imparts a significant risk factor for persistent pain and depression. Such an observation outlines a need to more carefully 
address pain management in the days following childbirth ${ }^{2}$. As the mother needs to bond with the new baby as early as possible and initiate breast feeding which helps to contract uterus and accelerates the process of uterine involution in the postpartum period. Effective treatment of postoperative pain contributes to decreasing the rate of complications as emotional and physical suffering, overt use of opioids, sleep disturbance , cardiovascular side effects, increased oxygen consumption as well as the total cost of the operated patients and development of chronic post surgical pain. Post caesarean section pain complicates the postoperative recovery in women $^{3 .}$

The fact that the pain comprises several components accounts for the necessity of multimodal analgesia technique to provide effective postop analgesia ${ }^{4}$. Intraperitoneal instillation of local anaesthetic has been promising to minimize postoperative pain. The local anaesthetic inhibits nociception by affecting nerve membrane associated proteins and by inhibiting the release of prostaglandins and other agents that sensitise or stimulate the nociceptors and contributes to inflammation. Instillation of intraperitoneal local anaesthetics lignocaine, bupivacaine, levobupivacaine and ropivacaine has been used following laparoscopic gynaecological and general surgical procedures to reduce pain through randomized trials for many years. ${ }^{5-10}$

The aim of our study was to evaluate the relative efficacy of Bupivacaine and Ropivacaine for postoperative analgesia in an elective caesarean section in relation to duration of postoperative analgesia and complications.

\section{Materials and Methods}

This randomised, prospective, double blind controlled study was carried out over a period from september 2017 to september 2018 after obtaining approval from Institutional Ethics Committee and written informed consent from the patients. Sample size was calculated using published data of a previous study based on the power of study. Power of study calculated using Open EPI version 2.3.1 software based on duration of analgesia.

90 ASA Grade I and II patients undergoing elective caesarean section with uncomplicated pregnancy of 18 to $30 \mathrm{yrs}$ of age were included in the study.

Parturients having pre-eclampsia, eclampsia, placenta previa or major illness, fetal compromise, patients with severe systemic illness, patients on analgesics and patients with history of allergy to local anaesthetics were excluded.

Each patients was randomly assigned to one of the three groups of 30 patients each using computer generated random table. Group Normal saline (C) received $30 \mathrm{ml}$ of $0.9 \%$ normal saline, Group Ropivacaine (R) received $30 \mathrm{ml}$ of $0.25 \%$ Ropivacaine (75mg) and Group (B) received 30 $\mathrm{ml}$ of $0.25 \%$ Bupivacaine. All the study drugs were prepared and coded by an anaesthetist who was not part of the study and anaesthetist conducting the study was unaware of the drug being injected. The patient was blind to the drug injected as well.

All patients underwent thorough medical evaluation and routine lab investigations a day prior to surgery. On the day of surgery after confirmation of fasting status the patient were shifted to OT and multipara monitors were attached. Baseline SBP, DBP, SPO2, pulse rate was recorded. I.V. line was accessed with two 20 $\mathrm{G}$ cannula. All patients received inj. Ranitidine 50 $\mathrm{mg}$ iv and inj. Metoclopramide $10 \mathrm{mg}$ iv for aspiration prophylaxis before surgery. After all routine monitoring of vitals having electrocardiography (ECG), Noninvasive blood pressure (NIBP), pulse oximetry (SPO2) under all aseptic precautions caesarean section were carried out under spinal anaesthesia using 25 Gauge Spinal needle. After incising all the layers of skin and subcutaneous tissue and separation of rectus muscle was done. Parietal peritoneum was opened and a small median transverse hysterotomy incision had been made and after delivery of the baby and the placenta, uterine closure, using 
continuous nonlocking double layer closure, without exteriorization was completed. As requested by the randomization code, patients received intraperitoneal instillation of either $30 \mathrm{cc}$ of sterile normal saline (0.9\%) (group C) or 30cc of ropivacaine $0.25 \%$ (group $\mathrm{R}$ ) using sterile syringes, so that the fluid was instilled into the pelvis. Vitals SBP, DBP, SPO2, pulse rate was recorded at 0 hrs .(T0). Care was taken that blood accumulating into the pelvis after closing the uterine incision and hematomas carefully wiped out with surgical towels leaving a more or less "dry" pelvis before the fluid was instilled. This was followed by closing the abdomen in different layers.

Follow up and assessment: After the completion of operative procedure pain was monitored at 0 hrs(T0) at the time of instillation subsequently 2(T1), 4(T2), 6(T3), 12(T4), 24(T5), 48(T6) hrs in the Post Anaesthesia Care Unit. VAS were performed to assess global abdominal pain and a mean pain score using VAS score was recorded at $0,2,4,6,12,24,48 \mathrm{hrs}$. As per requirement of the patient injection diclofenac intravenously as a form of rescue analgesia was given to maintain VAS $<6$ and the time was noted. The total analgesic requirement for $24 \mathrm{hrs}$. was recorded.

\section{Statistical analysis}

To collect required information from eligible patients a prestructured pre tested proforma was used. For data analysis microsoft excel and statistical software SPSS 22.0. Data were analysed with the help of percentage, mean, SD in the form of tables, diagrams. The results were statistiacally analysed by ANOVA test, used for quantitative data.

\section{Observation and Results}

There was an even distribution of age in all the groups. A random allocation of patients was done in different groups.The age distribution of the patients showed statistically insignificant changes. $(\mathrm{p}=0.064)($ Table 1)

\section{Distribution of age among the groups}

There was no significant difference in the hemodynamic parameters i.e. mean Pulse rate, MAP readings at all the time intervals.(Figure 12)

Table-1 Age Distribution in Different Groups

\begin{tabular}{|l|c|}
\hline & AGE (MEAN \pm SD) \\
\hline GROUP C & $25.36 \pm 2.12$ \\
\hline GROUP B & $24.00 \pm 2.59$ \\
\hline GROUP R & $24.23 \pm 2.40$ \\
\hline CD VALUE & 1.305 \\
\hline P value using F value & 0.064 \\
\hline
\end{tabular}

Table -2 Mean VAS in different groups

\begin{tabular}{|l|c|c|c|c|}
\hline $\begin{array}{l}\text { TIME } \\
\text { INTERVAL }\end{array}$ & GROUP C & GROUP B & GROUP R & CD VALUE \\
\hline T0(0 hrs.) & 0 & 0 & 0 & - \\
\hline T1(2 hrs.) & $5.16 \pm 1.34$ & $2.60 \pm 1.94$ & $1.0 \pm 1.80$ & 0.881 \\
\hline T2(4 hrs.) & $6.73 \pm 2.04$ & $4.86 \pm 2.55$ & $3.1 \pm 2.3$ & 1.190 \\
\hline T3(6 hrs.) & $3.83 \pm 1.87$ & $3.70 \pm 2.05$ & $3.56 \pm 2.02$ & 1.089 \\
\hline T4(12 hrs.) & $3.66 \pm 1.26$ & $2.76 \pm 1.85$ & $2.0 \pm 2.28$ & 0.952 \\
\hline T5(24 hrs.) & $2.0 \pm 0.91$ & $1.63 \pm 1.40$ & $0.6 \pm 0.93$ & 0.568 \\
\hline T6(48 hrs.) & $0.53 \pm 0.89$ & $0.267 \pm 0.69$ & $0.13 \pm 0.51$ & 0.393 \\
\hline
\end{tabular}


Figure-1 : Pulse Rate Variation

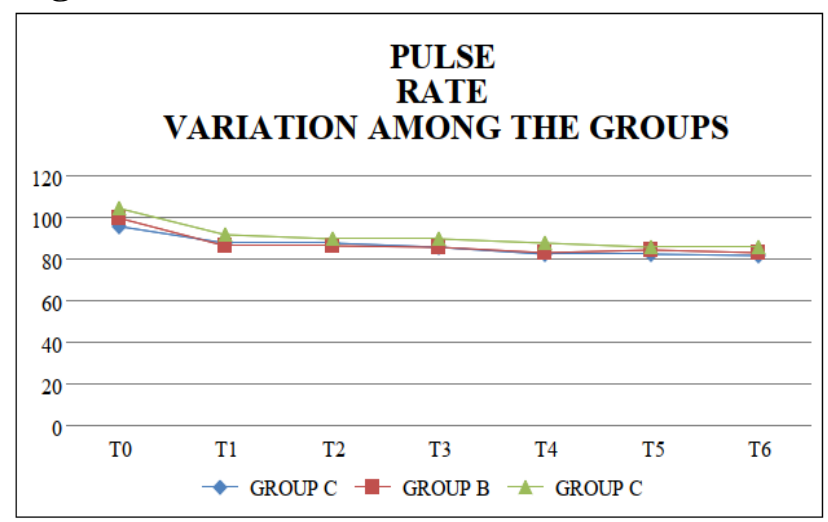

Figure -3 First dose of rescue analgesia

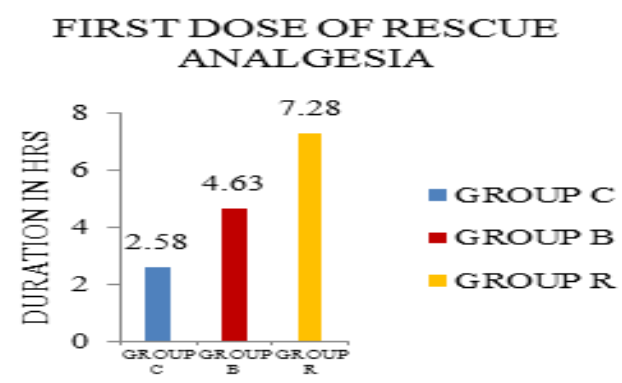

As shown in as shown in figure-1 the mean pulse rate was comparable among the groups. There is no significant variation in pulse rate in all the three groups. $(\mathrm{CD}$ value $>$ the difference of two mean).

As shown in figure-2 the Mean arterial blood pressure were comparable. The effect on Mean areterial blood pressure in all the groups found to be insignificant. (CD value $>$ the difference of two mean).

In our study we found that Ropivacaine group had low VAS score at all time intervals as comaparative to Bupivacaine and normal saline group except at $6 \mathrm{hrs}$. and $48 \mathrm{hrs}$. Time to first analgesic requirement was compared. The mean of duration of analgesia in Group $\mathrm{C}$ was $2.58 \pm 0.799$ hours, in Group B 4.63 \pm 1.04 hours and in Group $\mathrm{R} 7.28 \pm 3.36$ hours during the postoperative period. This difference was statistically as well as clinically significant.
Figure -2 Mean Blood Pressure Variation

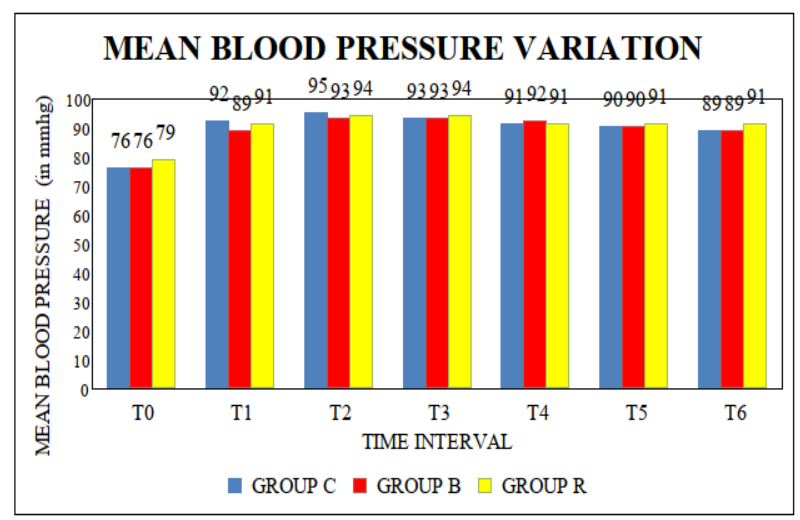

Figure-4 24 Hrs. Total Analgesic Consumption

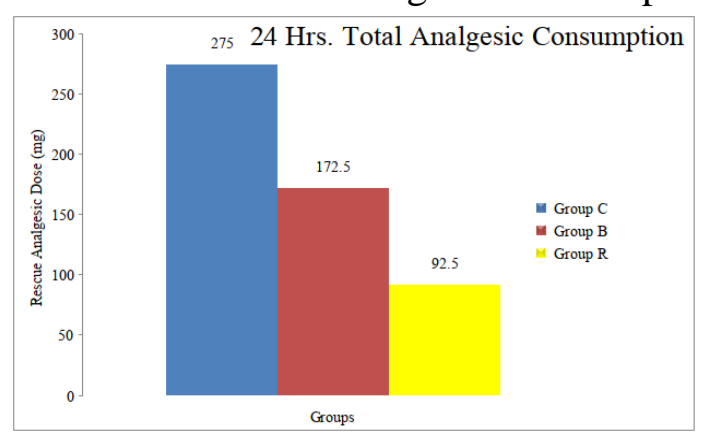

Table 3 Complication in Study Groups

\begin{tabular}{|c|c|c|c|}
\hline & NAUSEA & VOMITING & $\begin{array}{c}\% \\
\text { VALUE }\end{array}$ \\
\hline GROUP C & nil & nil & nil \\
\hline GROUP B & $2 / 30$ & nil & $6.6 \%$ \\
\hline GROUP R & $2 / 30$ & $2 / 30$ & $6.6 \%$ \\
\hline
\end{tabular}

The total analgesic consumption were observed in 3 different groups. In Ropivcaine group tthe total analgesic consumption was $92.50 \mathrm{mg} \pm 46.95$, Bupivacaine 172.5mg. \pm 40.12 and Control group 275mg. \pm 41.00 . Ropivacaine group has least rescue analgesic consumption which was statstically significant.

$6.6 \%$ patient complaint of only nausea in Group $\mathrm{B}$ and $6.6 \%$ patients complaint of nausea and vomiting both in Group R. This was found statistically and clinically insignificant.

\section{Discussion}

Surgical pain is due to inflammation from tissue trauma like surgical incision, dissection or direct nerve injury i.e. nerve transaction, stretching or compression. Various multimodal approaches have been tried to ameliorate postoperative pain. 
In the present study we compared the effect of intraperitoneal local anaesthetic drugs with normal saline. Donatsky AM et al. ${ }^{11}$ conducted a systematic review to study the role of intraperitoneal saline in prevention of postoperative pain in LC. Their findings also support the analgesic action of normal saline in the form of intraperitoneal instillation. There was an even distribution of age in all the groups. There was no significant difference in the hemodynamic parameters i.e. Pulse rate, SBP, DBP, MAP \& $\mathrm{SPO}_{2}$ readings at all the time intervals. In the present study, the mean postoperative systolic blood pressure, diastolic blood pressure and pulse rate readings were found to be lower in group $\mathrm{R}$ and were stable and comparable in all the groups. The reason being the rescue analgesia given on demand whenever VAS scores reached 6.The results were in concordance with a study done by Babu et al. ${ }^{12}$ where the vital parameters such as heart rate, blood pressure, and saturation were comparable between the groups.

In our study we found that Ropivacaine group had low VAS score at all time intervals as comparative to Bupivacaine and normal saline group except at $6 \mathrm{hrs}$. and $48 \mathrm{hrs}$. The reason being the rescue analgesia given on demand in Bupivacaine group and normal saline group and the longer duration of analgesic effect of ropivacaine in group R.Devalkar and Salgaonkar et al. ${ }^{13}$ found mean VAS score readings to be lower in Bupivacaine group as compared to normal saline group and were statistically significant 2, 4, 8 and $12 \mathrm{hr}$..

Our study showed that the analgesic effect was more pronounced in Ropivacaine group. Time to first analgesic requirement in group $\mathrm{C}$ was 2.58 hrs. \pm 0.799 , in group B 4.633 hrs. \pm 1.04 and group R 7.28 hrs. \pm 3.36 respectively. In ropivacaine group the pain was significantly reduced in immediate post-opertaive period till $8 \mathrm{hrs}$. as shown by the need of the first dose of rescue analgesic drug. The total analgesic requirement was less in Group $\mathrm{R}(92.50 \mathrm{mg} \pm 46.95)$ than Group B (172.5mg \pm 40.12) and Group C(275 mg \pm 41.00 ). This implies that the analgesia provided by Ropivacaine is of longer duration and denser than Bupivacaine. However in our study intraperitoneal instillation of both Ropivacaine and Bupivacaine reduced the pain. The above observation goes in concordance of other studies like Narchi $\mathrm{P}$ et al. ${ }^{14}$ compared intraperitoneal infiltration of $0.5 \%$ lignocaine and $0.125 \%$ bupivacaine with normal saline as control in day care laparoscopic surgeries.

We observed for various complications like nausea, vomiting, bradycardia, respiratory depression, hypotension and sweating in all patients. Complications were noted in $<10 \%$ of the patients in group B and group R. Nausea seen in 2 patients in group B. Nausea and vomiting seen in 2 patients in group $\mathrm{R}$. All the readings were comparable and the difference was found to be insignificant in group B an group R.This is favoured by a study done by Radhe Sharan et $\mathrm{al}^{15}$. They found that complications were noted in $<15 \%$ of the patients in their study groups.

There was no hypotension, arrhythmias or respiratory depression in any of the groups. This shows that intraperitoneal instillation of ropivacaine and bupivacaine in the volume and dose used in our study is not associated with any significant adverse effects.

\section{Conclusion}

The conclusion of this study is that intraperitoneal instillation of Ropivacaine can certainly be recommended as a routine technique to improve postoperative analgesia in an elective caesarean section.However larger randomized controlled trials are required to evaluate the efficacy and complications of intraperitoneal use of Ropivacaine.

\section{Bibliography}

1. Carvalho B, Cohen SE, Lipman SS, Fuller A, Mathusamy AD, Macario A(2005). Patient preference for anaesthesia outcomes with caesarean delivery. Anaesthesia and analgesia 2005;10 (14);1182-1187. 
2. Eisenach JC, Pan PH, Smiley R, Lavand' homme P, Landau R, Houle TT (2008). Severity of acute pain after childbirth but not type of delivery,predicts persistant pain and postpartum depression. Pain.2008 nov 15;140 (1) 87-94.

3. Luana Buhagiar, Olivia A Cassar, Mark P Brincat, George G Buttigieg, Anthony Serracino Inglott, Maurice Zarb Adami, Lilian M Azzopardi (2011) predictors of post caesarean section pain and analgesic consumption. J Anaesthesiol Clin Pharmacol 2011;27:185-91.

4. Goldstein Andrei; Grimault Patrick ; Henique Aude ; Keller Michèle ; Fortin Anne ; Darai Emile (2000). Preventing Postoperative Pain by Local Anesthetic Instillation After Laparoscopic Gynecologic Surgery: A Placebo-Controlled Comparison of Bupivacaine and Ropivacaine. Anesthesia \& Analgesia: August 2000 - Volume 91 - Issue 2 - p 403-407.

5. Roberts K, Gilmour J, Pande R, Nightingle P Tan L et al (2011). Efficacy of intraperitoneal local anaesthetic techniques during laparoscopic cholecystectomy. Surgical Endoscopy and Other Interventional Techniques 2011 nov;25 (11);3695-705.

6. Labaille, Thierry ; Mazoit, Jean Xavier ; Paqueron, Xavier ; Franco, Dominique Benhamou, Dan (2002). The clinical efficacy and pharmacokinetics of intraperitoneal ropivacaine for laparoscopic cholecystectomy. Anesthesia \& Analgesia: January 2002 - Volume 94 - Issue 1 - p 100-105

7. Sunil Chiruvella, Srinivasa Rao Nallam (2016). Intraperitoneal instillation of ropivacaine plus dexmedetomidine for pain relief after laparoscopic hysterectomy: A comparison with ropivacaine alone. J NTR Univ Health Sci 2016;5:93-7

8. Kucuk, C., Kadiogullari, N., Canoler, O., \& Savlı, S. (2007). A Placebo-Controlled Comparison of Bupivacaine and Ropivacaine Instillation for Preventing Postoperative Pain
After Laparoscopic Cholecystectomy. Surgery Today, 37(5), 396-400.

9. Ahmed BH, Ahmed A, Tan D, Awad ZT, AlAali AY, Kilkenny J, et al. Post-laparoscopic cholecystectomy pain: effects of intraperitoneal local anaesthetics on pain control - a randomized prospective doubleblinded placebo-controlled trial. Am Surg. 2008;74(3):201-09.

10. Saad Ei Basha, Maha G Hanna, Sherif M Soaida, Hagar H Rafaee. Intraperitoneal instillation of 1-bupivacaine in laparoscopic pediatric procedures: a randomized controlled study. Ain-Shams Journal of Anaesthesiology8(2), 247, 2015.

11. Donatsky AM, Bjerrum F, Gögenur I. Intraperitoneal instillation of saline and local anaesthesia for prevention of shoulder pain after laparoscopic cholecystectomy: a systematic review. Surg Endosc. 2013;27(7):2283-92.

12. Babu R, Jain P, Sherif L. Intraperitoneal instillation: ropivacaine vs bupivacaine for post operative pain relief in laparoscopic cholecystectomy. Int J Health Sci Res. 2013;3(12):42-47.

13. Devalkar PS, Salgaonkar SV. Intraperitonneal instillation of $0.25 \%$ bupivacaine for laparoscopic cholecystectomy: Effect on postoperative pain. IJCMAAS.2016;12;91-5.

14. Narchi P, Benhamou D, Fernandez H. Intraperitoneal local anaesthetic for shoulder pain after day-case laparoscopy. Lancet. 1991;338:1569-70.

15. Sharan R, Singh M, Kataria AP, Jyoti K, Jarewal V, Kadian R. Intraperitoneal Instillation of Bupivacaine and Ropivacaine for Postoperative Analgesia in Laparoscopic Cholecystectomy. Anesth Essays Res. 2018;12(2):377-380. 